\title{
Nuclear Import Analysis of Two Different Fluorescent Marker Proteins into Hepatocyte Cell Lines (HuH-7 Cell)
}

\author{
Aris Haryanto ${ }^{1^{*}}$ and Michael Kann ${ }^{2}$
}

1. Department of Biochemistry, Faculty of Veterinary Medicine, Gadjah Mada University. Jl. Olahraga, Karangmalang, Yogyakarta 55281, Indonesia.

2. Institute of Medical Virology, Justus-Liebig-University, Giessen, Germany. Frankfurterstrasse 107, 35392 Giessen, Germany

\begin{abstract}
s
The application of fluorescent proteins as expression markers and protein fusion partners has proved immensely valuable for resolving the organization of biological events in living cells. EGFP and DsRed2 are commonly fluorescent marker protein which is used for biotechnology and cell biology research. The present study was designed to identify the expression vector that suitable to ligate with DNA encoding HBV core protein for intracellular localization study in hepatocyte cell, which were expressed as fusion proteins. We also compared and quantified the expressed fluorescent protein which predominantly localized in the cell compartment. The results indicated that DsRed2 shown as less than ideal for intracellular localization study of than EGFP, because of its tetrameric structure of the fluorescent protein and when fused to a protein of interest, the fusion protein often forms aggregates in the living cells. In contrast, EGFP fluorescent protein shown a much higher proportion of cytoplasmic localization, thus being more suitable for analysis of intracellular localization than DsRed2 fluorescent protein. EGFP fluorescent protein is also capable to produce a strong green fluorescence when excited by blue light, without any exogenously added substrate or cofactor, events inside living cell can thus be visualized in a non-invasive way. Based on our present quantitative data and some reasons above shown that EGFP is more suitable than DsRed2 as a fluorescent marker protein for intracellular localization study into HuH-7 cell.
\end{abstract}

Keywords: EGFP, DsRed2 fluorescent protein , HuH-7 cell, HBV, intracellular localization

\section{Introduction}

The enhanced green fluorescent protein (EGFP) is a marker protein that comprised of 238 amino acids with molecular weight $27 \mathrm{kDa}$. This marker protein was isolated from the jellyfish Aequorea victoria which fluoresces green color when exposed to blue light (Prendergast and Mann, 1978; Tsien, 1998). The EGFP gene is frequently used as a reporter expression (Philip, 2001). In modified forms it has been used to make biosensors, and many animals have been created that express EGFP as a proof of

\footnotetext{
*Corresponding author: Aris Haryanto, Department of Biochemistry, Faculty of Veterinary Medicine, Gadjah Mada University, Jl.Olah Raga Karangmalang, Yogyakarta 55281, Indonesia. Tel: 62-274-560865; Email: arisharyanto@yahoo.com
}

concept that a gene can be expressed throughout a given organism. The availability of EGFP has thoroughly redefined fluorescent and the way it is used in cell biology and other biological disciplines (Yuste, 2005).

EGFP are usually much less harmful when illuminated in living cells. This has triggered the development of highly automated live cell fluorescence microscopy systems which can be used to observe cells over time expressing one or more proteins tagged with fluorescent proteins. Another powerful using of EGFP is to express the protein in small sets of specific cells. This allows researchers to optically detect specific types of cells in vitro or even in vivo 
(Chudakov, 2005). The EGFP gene can be introduced into organisms and maintained in their genome through breeding, or local injection with a viral vector can be used to introduce the gene. Currently, many bacteria, yeast and other fungal cells, plant, fly, and mammalian cells have been created using EGFP as a protein marker.

DsRed is the first coral-derived fluorescent marker protein which to be extensively utilized for biotechnology and cell biology as a spectrally distinct companion or substitute for the green fluorescent protein (GFP) from the Aequorea jellyfish. It was derived from Discosoma striata (Matz et al., 1999). Wild type DsRed is an obligate tetramer. When fused to a protein of interest, the fusion protein often forms aggregates in living cells, hampering normal localization, trafficking and proteinprotein interactions of the protein of interest. Although these features are inconsequential for the use of DsRed as a reporter of gene expression, the usefulness of DsRed as an epitope tag is severely limited. In contrast, to the jellyfish fluorescent proteins, which have been successfully used to tag hundreds of proteins, DsRed conjugates have proven much less successful and are often toxic. The evolution of DsRed from a scientific curiosity to a generally applicable and robust tool has been hampered by several critical problems, including a slow and incomplete maturation and obligate tetramerization (Baird et al., 2000).

The second generation of DsRed is called DsRed2. This fluorescent marker protein contains several mutation at the peptide amino terminus which prevent formation of protein aggreates. The mutation also reduce toxicity effect to the transfected cell. As DsRed, the fluorescent marker protein of DsRed2 still forms a tetramer. Red fluorescence emission from DsRed2 can be observed approximately 6 hours after transfected. It is faster than DsRed which can be observed after 11 hours posttransfection.

The present study was designed to identify the expression vector that suitable to ligate with DNA encoding HBV core protein for intracellular localization study of HBV core protein into hepatocyte cell, which were expressed as fusion proteins. We also compared and quantified the expressed fluorescent protein which predomonantly localized in the cell compartment.

\section{Materials and Methods}

Main materials of this work are plasmid vector encoding red fluorescent marker protein pDsRed2-C1 (Clonetech), plasmid vector encoding green fluorescent marker protein pEGFP-C3 (Clonetech) and hepatocyte cell line $\mathrm{HuH}-7$.

\section{Preparation of $\mathrm{pDsRed2-C1}$ and $\mathrm{pEGFP}-$ C3}

To clone plasmid vector encoding red fluorescent and green fluorescent marker protein, pDsRed2-C1 and pEGFP-C3 were transformed into competent E. coli XL-1 blue. Into each $1.5 \mathrm{ml}$ E-cup, $1 \mu \mathrm{l}$ DNA pDsRed2C1 and pEGFP-C3 were added into $25 \mu$ competent $E$. coli. After gentle mixing, the transformation reactions were cooled down on the ice for $10 \mathrm{~min}$, then the transformation was accomplished by a heat shock at $42^{\circ} \mathrm{C}$ for $2 \mathrm{~min}$. Afterwards $1 \mathrm{ml}$ LB medium was added and mixed gently. The E. coli were incubated at $37^{\circ} \mathrm{C}$ for $1 \mathrm{~h}$ and platted onto an agar dish containing LB medium with $30 \mathrm{mg} / \mathrm{ml}$ Kanamycin. The dish was incubated at $37^{\circ} \mathrm{C}$ until the liquid has been absorbed. Then the dish was inverted and incubated over night at $37^{\circ} \mathrm{C}$.

Maxipreparation DNA plasmid isolation After $16 \mathrm{~h}$, the bacterial colonies appeared. Using sterile yellow tips, the bacterial colonies were picked and grown 
in $250 \mathrm{ml}$ Kanamycin $(30 \mathrm{mg} / \mathrm{ml})$ containing LB medium. The colonies were incubated in shaking incubator at $37^{\circ} \mathrm{C}$ overnight. The next day, $250 \mathrm{ml}$ bacteria in LB medium was harvested and sedimented. The plasmid DNA was isolated by a Maxipreparation DNA plasmid isolation kit (Qiagen). This plasmid isolation was performed according to the manufacture recommendations.

\section{Preparation of hepatocyte cell line (HuH- 7 cell)}

Hepatocyte cell lines (HuH-7 cells) were seeded on the culture dish and incubated over night in the incubator $\mathrm{CO}_{2}$ at $37^{\circ} \mathrm{C}$. The growing cells were harvested and washed with PBS. The PBS was replaced by $1 x$ trypsin in PBS (Gibco-BRL), which has been preincubated at $37^{\circ} \mathrm{C}$. While the dish with the cells was incubated at $37^{\circ} \mathrm{C}$ for $2-5 \mathrm{~min}$, $0.5 \mathrm{ml} 10 \%$ FCS containing medium (GibcoBRL) were added to the each well of 24 well dish containing collagen-treated cover slips (Nunc Inc.). The trypsin in PBS was removed and washed the cells with PBS. The PBS was changed with fresh 10\% FCS containing medium. Each cover slip were filled with 2-5 drops cell suspension. Then the cells were allowed to grow over night at $37^{\circ} \mathrm{C}$ in humidified incubator at $5 \% \mathrm{CO}_{2}$.

\section{Transfection into HuH-7 Cell}

For transfection, Tfx-20 (Promega) was used as transfection agent, $1 \mathrm{ml}$ DNA (1 $\mathrm{mg} / \mathrm{ml}$ ) of pDsRed2-C1 and pEGFP-C3 were mixed gently with $3 \mathrm{ml} \mathrm{Tfx}-20$, then added into $300 \mathrm{ml}$ FCS-free medium (GibcoBRL). The mixture was incubated at room temperature for 5-10 min. During the incubation, the medium in the 24 well dish were removed and replaced with FCS free medium. The DNA/Tfx-20 reagent/FCSfree medium mixture were vortexed briefly, then added the mixture to the cells $(300 \mathrm{ml}$ per well) and incubated in the incubator at
$5 \% \mathrm{CO}_{2}$ and $37{ }^{\circ} \mathrm{C}$ for $1 \mathrm{~h}$. During the incubation $10 \%$ FCS containing medium was warmed at $37^{\circ} \mathrm{C}$ in the waterbath. After $1 \mathrm{~h}$ the FCS free medium was replaced with the $10 \%$ FCS containing medium and the dish was returned into the incubator for 24 $\mathrm{h}$.

\section{Indirect immune fluorescence staining}

$\mathrm{HuH}-7$ cells were fixed on the cover slips by 3\% paraformaldehyd (PFA) (Sigma) in PBS at room temperature for $30 \mathrm{~min}$. After fixation the cells were washed with 1x PBS three times. Then the cells were treated with $500 \mathrm{ml} 0.1 \%$ Triton X-100 (Serva). The dish was incubated at room temperature for $10 \mathrm{~min}$ in order to destroy the cell membranes. After that, the wells were washed three times with $1 x$ PBS. For transformed $\mathrm{HuH}-7$ cells by pEGFP-C3, indirect immune fluorescence staining were performed using primary antibody mixture containing monoclonal antibody (mAb) 414 anti NPC (BabCo) from mouse ( 1: 300 dilution) in antibody solution (1x PBS / 5\% BSA/ $5 \%$ goat serum). For each cover slip $40 \mu \mathrm{l}$ antibody mixture spotted on parafilm in a humidified box. The cover slips were placed with their cell side on the drop of antibody mixture. The humidified box was incubated at $37^{\circ} \mathrm{C}$ in the incubator. After 1 $h$, the up side of cover slips were rinsed with $100 \mathrm{ml} \mathrm{1x}$ PBS before replaced in the 24well dish. The 24 well dish was washed three times with 1x PBS. For secondary antibody reaction a goat Texas Red-labelled anti mouse antibody (Dianova) were diluted 1: 100 in antibody solution. $40 \mathrm{ml}$ this antibody mixture was spotted on parafilm in the humidified box followed by transfer of the cover slips as described before. The box was incubated at $37^{\circ} \mathrm{C}$ for $45 \mathrm{~min}$, the cover slips were rinsed, replaced and washed as described. Drops of DabcoMoviol (Sigma-Hoechst) were spotted on microscopical glass slides and the cover slips 
were placed on them. The glass slides were kept over night in the darkness at room temperature. For transformed HuH-7 cells by pDsRed2-C1, indirect immune fluorescence staining were performed using primary antibody mixture containing monoclonal antibody (mAb) 414 anti NPC from mouse (1: 300 dilution) and as secondary antibody used goat Alexa 488labelled anti mouse antibody (Dianova). Immune fluorescence procedure was performed as described above.

\section{Immune fluorescence confocal laser microscopy}

A drop of immersion oil was dripped on each of cover slips in the slide. The slide was put on the microscope. The cells were analyzed using a $63 x$ apochromat objective by a LEICA DM IRBE. For the confocal analysis the cells were scanned by a laser power of $30 \%$, a signal amplification of $85 \%$ and a pinhole size of 0.9 with a two-fold magnification. Eight pictures were merged. For the representation of Alexa 488, the FITC filter settings were used, for depicting Texas Red, the TRITC filter setting were used. The $\mathrm{z}$ position in all picture was adjusted to the equatorial level of the nuclei. The pictures were arranged using the ADOBEPHOTOSHOP software program.

\section{Quantification of the Intracellular Localization}

Red fluorescent and green fluorescent marker protein which found localized in the compartment of $\mathrm{HuH}-7$ cells, was quantified manually using confocal laser microscope. The Amount of fluorescent proteins that found localized in the cytoplasm, nucleus or both of cell compartment were quantified in the absolute and relative values.

\section{Results and Discussion}

Analysis expression of the fluorescent proteins by E. coli transformed with plasmids encoding EGFP and DsRed2 marker protein could clearly analyze in the transfected $\mathrm{HuH}-7$ cells $12 \mathrm{~h}$ after transfection for efficient visualization using confocal laser scan microscope. In the cells expressing EGFP, the fluorescent proteins found localized in the cytoplasm, nucleus and both of the cell compartment (Figure 1). In contrast DsRed fluorescences found distributed only in cytoplasm and nucleus (Figure 2). It might indicate that EGFP fluorescent protein which monomer form can passively imported into the nucleus, even very rare and only minority amount. Whereas DsRed2 fluorescent protein can not passive imported into the nucleus because this fluorescent protein is tetramer with molecular weight higher than EGFP fluorescent protein.

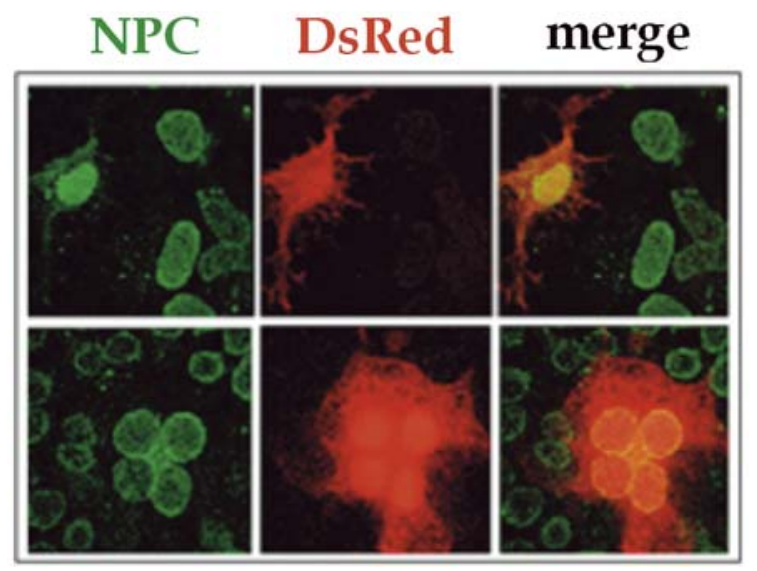

Figure 1. Intracellular Localization of DsRed2 marker protein in HuH-7 cells. DsRed2 (middle column) was found to be localized inside the cytoplasm (upper row) and within the nucleus (lower row) in a similar amount of cells. The nuclear pore complexes are shown in green; the overlays (merge) in the right column.

To investigate the intracellular localization of two commonly used fluorescent marker proteins, DsRed (red 
fluorescence) and EGFP (green fluorescence) by transient expression of the corresponding expression vectors $\mathrm{pDsRed} 2-$ $\mathrm{C} 1$ and pEGFP-C3 in HuH-7 cells (Figure 1 and 2). Both fluorescent marker proteins were expressed under the control of the cytomegalovirus promoter $\left(\mathrm{P}_{\mathrm{CMV}}\right)$. The transfection and cell culture conditions were identical to those used for the expression of the HBV capsid in the experiment before. As in this experiment, the staining of the NPCs was done using the same primary antibody. In pDsRed 2 transfection an FITC-labelled secondary antibody was used. For localisation of EGFP the secondary antibody was Texas Red labelled. Both transfections were done in parallel to exclude differences caused by the pre-cultivation of the cells prior to transfect into $\mathrm{HuH}-7$ cells.
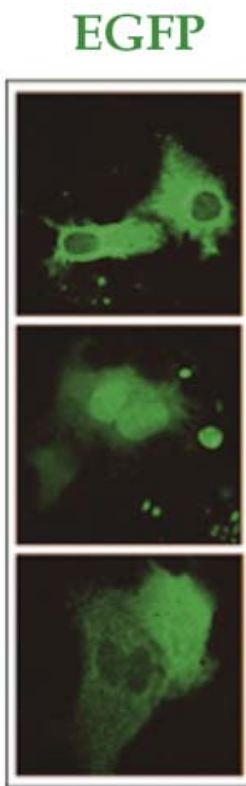

NPC
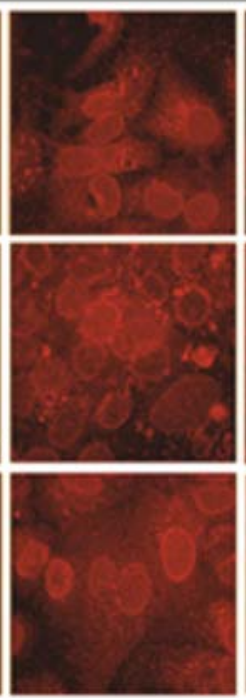

\section{merge}

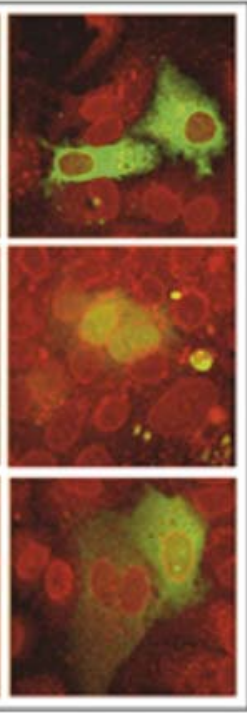

Figure 2. Intracellular Localization of EGFP marker protein in HuH-7 cells. In the majority of cells EGFP (green) localized predominantly in the cytoplasm (upper row). In a minority of cells EGFP was localized nucleus (midlle row) or throughout cytoplasm and nucleus (lower row, right cell). The nuclear pore complexes are shown in red; the overlays (merge) in the right column.

Absolute and relative numbers of fluorescent marker protein distribution in the cell compartment showing an equal distribution or a predominantly cytoplasmic or nuclear localization of the fluorescent proteins (Tabel 1.). Both transfections of DsRed 2 and EGFP fluorescent proteins were performed in parallel work.

Tabel 1. Distribution of DsRed and EGFP in HuH-7 cells after transient expression into $\mathrm{HuH}-7$ cells.

\begin{tabular}{lcccc}
\hline marker & cytoplasm & nucleus & both & total \\
\hline DsRed2 & 7 & 10 & 0 & 17 \\
\hline & $41 \%$ & $59 \%$ & 0 & $100 \%$ \\
\hline EGFP & 100 & 39 & 1 & 140 \\
\hline & $71 \%$ & $28 \%$ & $1 \%$ & $100 \%$ \\
\hline
\end{tabular}

A proportion of both proteins localized inside the nucleus. In both cellular compartments both of fluorescent proteins were distributed disperse, showing no association to cellular structures. Distribution of EGFP fluorescent proteins were only rarely found in both, cytoplasm and nucleus indicating that they are not freely diffusing. The significant number of cells showing a nuclear fluorescence however indicates that either both proteins are weakly karyophilic or that they are passively trapped upon reconstitution of the nuclear membrane following cell division. Since DsRed form tetramers with a resulting molecular weight of $89 \mathrm{kDa}$, a passive trapping upon cell division appears more likely assuming a diffusion limit of approximately $40-45 \mathrm{kDa}$ through the nuclear pores (Dingwall and Laskey, 1992). Some properties of DsRed were also recognized as less than ideal for biological applications, such as the tetrameric structure of the protein and the propensity of tetramers to oligomerize further into aggregates impeded its utility as a fusion 
partner, and the complex fluorophore maturation kinetics via a green fluorescent intermediate were tardy (half-time; 1 day), incomplete, and spectrally inconvenient for multicolor labeling applications, in which cross talk with GFP-like proteins remained a problem, even after prolonged maturation (Baird et al., 2000; Gross et al., 2000; Mizuno et al., 2001).

EGFP fluorescent protein shows a much higher proportion of cytoplasmic localization, thus being more suitable for analysis of intracellular localization than DsRed2 fluorescent protein. However, the significant proportion of nuclear EGFP fluorescent protein showed that determination of the ratio of cells showing nuclear and/or cytoplasmic fusion protein is required. EGFP fluorescent protein shown a powerful tool for identifying the intracellular localization of fusion protein and also to monitor gene expression. This marker protein is capable of producing a strong green fluorescence when excited by blue light, without any exogenously added substrate or cofactor, events inside living cell can thus be visualized in a non-invasive way (Chalfie et al., 1994; Mayer et al., 1999; Niedenthal et al., 1996). Based on our present quantitative data and some reasons above shown that EGFP is more suitable than DsRed2 as a fluorescent marker protein for intracellular localization study in the HuH-7 cell.

\section{Acknowledgements}

The authors wish to express gratitude to Prof. Dr. Wolfram H. Gerlich, the Head Institute of Medical Virology, Justus-LiebigUniversity Giessen, Germany for the opportunity to do this work. This research was supported by Sonder Forschung Bereich (SFB) 535, Justus-Liebig-University Giessen, Teilproject B-6 (AG Kann) and Deutsche Akademische Austauschdienst (DAAD) Referat 422.

\section{References}

Baird, G. S., Zacharias, D.A., and Tsien, R.Y., 2000. Biochemistry, mutagenesis, and oligomerization of DsRed, a red fluorescent protein from coral. Proc. Natl. Acad. Sci. USA., 97, 1198411989.

Chalfie, M., Tu, Y., Euskirchen, G., Ward, W.W., and Prasher, D.C., 1994. Green fluorescent proteoin as marker for gene expression. Science, 263, 802-803.

Chudakov, D., Lukyanov, S., and Lukyanov K., 2005. Fluorescent proteins as a toolkit for in vivo imaging. Trends Biotechnol., 23 (12), 605-13.

Dingwall, C. and Laskey, R., 1992. The nuclear membrane. Science, 258, 942947.

Gross, L.A., Baird, G.S., Hoffman, R.C., Baldridge, K.K., and Tsien, R.Y., 2000. The structure of the chromophore within DsRed,a red fluorescent protein from coral. Proc. Natl. Acad. Sci. USA., 97, 11990-11995.

Matz, M.V., Fradkov, A.F., Labas, Y.A., Savitsky, A.P., Zaraisky, A.G., Markelov, M.L., and Lukyanov, S.A., 1999. Fluorescent proteins from nonbioluminescent Anthozoa species. Nat. Biotechnol., 17, 969-973.

Mayer, G., Launhardt, H., and Munder, T., 1999. Application of the green fluorescent protein as a reporter for AceI-based, two hibrid studies. BioTechniques, 27, 84-86.

Mizuno, H., Sawano, A., Eli, P., Hama, H., and Miyawaki,A., 2001. Red fluorescent protein from Discosoma as a fusion tag and a partner for fluorescence resonance energy transfer. Biochemistry, 27, 2502- 2510.

Niedenthal, R.K., Riles, L., Johnston, M., and Hegemann, J.H., 1996. Green fluorescent protein as a marker for gene expression and subcellular 
localization in budding yeast. Yeast, 12, 773-786.

Phillips, G., 2001. Green fluorescent protein - a bright idea for the study of bacterial protein localization. FEMS Microbiol. Lett., 204 (1), 9-18.

Prendergast, F. and Mann, K., 1978. Chemical and physical properties of aequorin and the green fluorescent protein isolated from Aequorea forskålea. Biochemistry, 17 (17), 344853.

Tsien, R.Y., 1998. The green fluorescent protein. Annu. Rev. Biochem., 67, 509544.

Yuste, R., 2005. Fluorescence microscopy today. Nat. Methods, 2 (12), 902-4. 